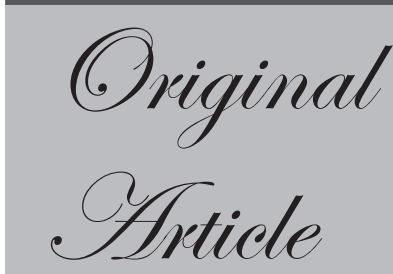

${ }^{I}$ Department of GI Sciences, Christian Medical College and Hospital, Vellore-632004, India

${ }^{2}$ Institute of Gastroenterology and Liver Disease, The Madras Medical Mission-600037, Chennai , India

Corresponding Author: Dr. Deepu David Email: deepudavid@gmail.com

\section{Endoscopic Ultrasonography Reveals Occurrence of Chronic Pancreatitis in Patients with Idiopathic Recurrent Acute Pancreatitis}

\author{
Reuben Thomas Kurien ${ }^{1}$, Deepu David ${ }^{1}$, Kattiparambil \\ Gangadharan Sajith ${ }^{1}$, Anjilivelil Joseph Joseph ${ }^{1}$, \\ Sudipta Dhar Chowdhury ${ }^{1}$, Ebby George Simon ${ }^{1}$, \\ Bharath Kumar Chandrashekaraiah ${ }^{1}$, Bharath Kumar \\ Ayapati $^{1}$, Rajeev SA ${ }^{1}$, Kaushik Chatterjee ${ }^{1}$, Ashok \\ Chacko $^{2}$, Amit Kumar Dutta ${ }^{1}$
}

\section{ABSTRACT}

Background: Chronic pancreatitis develops in a subgroup of patients with recurrent acute pancreatitis. Detection of chronicity helps prognostication and formulation of management plan. Endoscopic ultrasonography (EUS) may detect early stages of chronic pancreatitis and hence serve as a useful diagnostic tool.

Aim: To assess the frequency of chronic pancreatitis in patients with idiopathic recurrent acute pancreatitis (IRAP) using EUS.

Methods: Patients with diagnosis of IRAP over a ten year period were retrospectively included. The clinical and investigation details were recorded on a structured proforma. Details of findings on EUS were recorded and proportion of patients with chronic pancreatitis was estimated. Presence of calcification and eight other parenchymal/ductal features were assessed during EUS. Presence of calcification or five of the other eight features were required for the diagnosis of chronic pancreatitis.

Results: 154 patients with IRAP were included in the study. Their mean age was $31.2+12.5$ years and $77.9 \%$ were male. Thirtyeight $(24.7 \%)$ patients had definitive features of chronic pancreatitis on EUS. Another 54 (35.1\%) patients with 3 or 4 criteria present were considered to have intermediate probability of chronic pancreatitis. Calcification was present in 19 patients which was not detected on transabdominal imaging (ultrasound or CT). Twenty-two patients had diabetes mellitus.

Conclusion: EUS detected features of chronic pancreatitis in a quarter of patients with IRAP. Hence EUS may play an important role in management of these patients.

KEYWORDS: Pancreatitis, Chronic, IRAP, Endoscopic ultrasound. 


\section{Introduction}

Up to one-third of patients with acute pancreatitis develop recurrent episodes in the future. ${ }^{1}$ Despiteappropriate evaluation of these patients, the etiology is not apparent in $10-30 \%$ of them and they are labelled as having idiopathic recurrent acute pancreatitis (IRAP). ${ }^{2}$ While there are suggestions that some of these patients with IRAP may have underlying chronic pancreatitis, the data on actual frequency of chronicity is limited., ${ }^{3,4}$ Conventional imaging modalities like transabdominal ultrasound and computed tomography have poor sensitivity in detecting early stages of chronic pancreatitis. Endoscopic ultrasonography (EUS) is able to detect early changes of chronic pancreatitis and has good correlation with histology and pancreatic function tests. ${ }^{5,6}$ The availability and popularity of endoscopic ultrasonography have provided us with an opportunity for better understanding, early diagnosis and improved management of pancreatic disorders. In this paper, we report our findings of EUS in patients with IRAP along with the prevalence of chronic pancreatitis in them.

\section{Materials and Methods}

Thisobservational study retrospectively included patients with recurrent acute pancreatitis seen at our center from 2006 to 2015. Acute pancreatitis was diagnosed when epigastric pain occurred in association with elevated pancreatic enzymes (serum amylase or lipase more than 3 times the upper limit of normal) and/or imaging features suggestive of pancreatic inflammation. Recurrent acute pancreatitis was defined as occurrence of two or more such episodes at least 2 months apart7. All patients underwent an ultrasonography or CT scan of the abdomen.Those with imaging features of chronic pancreatitis at the time of initial diagnosis were excluded. Patients with recurrent acute pancreatitis underwent extensive evaluation for underlying etiology. This included evaluation for significant alcohol intake, gall stones (transabdominal ultrasonography and EUS), common bile duct stones (EUS), hypercalcemia (serum calcium $>10.5 \mathrm{mg} / \mathrm{dl}$ ), hypertriglyceridemia (serum triglyceride $>500 \mathrm{mg}$ / dl), biliary microliths (sludge on EUS or microliths on microscopic examination of unstimulated duodenalbile), antinuclear antibody, drug intake, abdominal trauma, pancreas divisum (excluded if EUS showed 'stack sign' or main pancreatic duct could be traced from the major papilla through the genu to the body of the pancreas), pancreatic malignancy (EUS), annular pancreas (EUS), parasite(normal bile duct on EUS) and choledochal cyst. ${ }^{2,4,8}$ If no cause could be ascertained after complete evaluation (including EUS), the recurrent acute pancreatitis was considered idiopathic (IRAP). ${ }^{9}$

The clinical and investigation details as well as findings on EUS among patients with IRAP were recorded on a uniform data sheet. The presence of parenchymal and ductal features suggestive of chronic pancreatitis on EUS was analysed. The parenchymal features included presence of hyperechoic foci, hyperechoic strands, hypoechoic lobules and parenchymal cysts. ${ }^{10}$ Ductal criteria included dilated main pancreatic duct $(>3 \mathrm{~mm}$ in head,or $>2 \mathrm{~mm}$ in body or $>1 \mathrm{~mm}$ in tail), irregular duct margin, hyperechoic duct margin, dilated side branches and presence of stones. ${ }^{10,11}$ Patients with five or more of the above criteria were considered to have chronic pancreatitis. ${ }^{4,10}$ Those with three or four features were considered to have intermediate probability of chronic pancreatitis. ${ }^{11}$ Patients with pancreatic stones in the setting of recurrent epigastric pain were also diagnosedto have chronic pancreatitis even if they had less than five criteria. ${ }^{11}$ Informed consent was obtained from all the patients prior to the procedure and the procedure was performed under conscious sedation using linear orradial EUS probe (Olympus or Pentax). Patients undergoing EUS within 4 weeks of an episode of acute pancreatitis were excluded as some of the EUS features of acute pancreatitis may overlap with those seen in chronic pancreatitis. ${ }^{11}$

Continuous data are presented as mean with standard deviation. Categorical data are summarised in percentages. Data analysis was done using SPSS v13 for Microsoft Windows.

\section{Results}

One hundred and fifty four patients with IRAP were included in the study. Their mean age was $31.2+12.5$ years and $77.9 \%$ were male. Diabetes mellitus was 
present in 22(14.3\%) of them. Laboratory evaluation showed a mean blood haemoglobin level of $13+1.9 \mathrm{~g} /$ $\mathrm{dl}$ and mean albumin level of $4.6+0.4 \mathrm{~g} / \mathrm{dl}$. Magnetic resonance cholangiopacreatography(MRCP) was done in twenty five patients. None of the patients had evidence of pancreas divisum on MRCP.

EUS revealed features of chronic pancreatitis in $38(24.7 \%)$ patients. These patients had $>5$ criteria of chronic pancreatitis or had calcifications/stone. Another $54(35.1 \%)$ patients with presence of 3 or 4 criteria and absence of calcification were considered to have intermediate probability of chronic pancreatitis. In summary, $24.7 \%$ patients had definitive features of chronic pancreatitis and $35.1 \%$ had intermediate probability of chronic pancreatitis on EUS.

Table 1 shows the frequency of individual parenchymal and ductal features suggestive of chronic pancreatitis among the study patients. Parenchymal features were commoner than ductal features except for the low frequency of cysts. Hyperechoic foci and strands were the commonest findings. Hyperechoic margin of the main pancreatic duct was the most frequent of the ductal criteria. Calcification/stone were seen in 19 (12.3\%) patients which was diagnostic of chronic pancreatitis. Among the 22 diabetics, 16 (72.7\%) showed features of chronic pacreatitis, where10patients had intermediate probability and 6 had definitive features of chronic pancreatitis. This is an increased proportion when compared to non diabetics, where $57.6 \%$ showed features of chronic pacreatitis. The difference was however not statistically significant $(\mathrm{p}=0.24)$.

Nineteen patients had calcification in the pancreas which was considered diagnostic of chronic pancreatitis. Sixteen of them had five or more features on EUS examination and the remaining three had three or four features. Hence, even though calcification alone is diagnostic of chronic pancreatitis, it is usually accompanied by other ductal and parenchymal features of chronic pancreatitis on EUS.

\section{Discussion}

Evaluation of patientswithrecurrent pancreatitis is challenging. When the etiology is elusive, the prognosis
Table 1: EUS findings in patients with IRAP

\begin{tabular}{l|l} 
Feature & Frequency $(\mathbf{n}=\mathbf{1 5 4})$ \\
\hline Hyperechoic Foci & $82.5 \%$ \\
\hline Hyperechoic Strands & $77.3 \%$ \\
\hline Hyperechoic Lobules & $41.6 \%$ \\
\hline Cysts & $1.3 \%$ \\
\hline Hyperechoic MPD Margin & $39 \%$ \\
\hline Dilated MPD & $17.5 \%$ \\
\hline Irregular MPD & $16.2 \%$ \\
\hline Visible Side Branches & $5.2 \%$ \\
\hline Calcification / Stone & $12.3 \%$ \\
\hline
\end{tabular}

and future management strategyis unclear. ${ }^{12}$ EUS has proven to be a very useful modality in management of this group of patients. ${ }^{13}$ It provides high resolution, real time imaging of the pancreas and biliary system which is not hampered by bowel gas. In addition to identifying etiological factors like microlithiasis, pancreas divisum, pancreatic neoplasms, etc., it is able to detect early stages of chronic pancreatitis which may be missed on other imaging modalities. ${ }^{13,14}$ In our study which included a large number of patients with IRAP, EUS revealed intermediate or high probability of chronic pancreatitis in about $60 \%$. This significantly alters their management and prognosis. These patients may benefit from pancreatic enzyme supplements, low fat diet, antioxidants, etc. In terms of prognosis, patients with chronic pancreatitis have higher mortality rate than the general population. In addition, these patients have to be kept under regular follow up as complications like diabetes, steatorrhea, pseudocyst or malignancy may develop in future. ${ }^{15}$ Previous studies have shown the prevalence of chronic pancreatitis to range from $10-40 \%$ in patients with idiopathic acute pancreatitis. ${ }^{3,4,16,17}$ However, these studies were limited by small sample sizes(except for the study by Yusoff et al.) andinclusion of all patients with idiopathic acute pancreatitis (one or recurrent episodes). ${ }^{3,4}$

About $40 \%$ of our patients did not have sufficient features for diagnosing chronic pancreatitis with intermediate or high probability. It is possible that some of them may develop features of chronic pancreatitis in future and they need to be kept under surveillance. Follow up data of these patients as well as those with intermediate 
probability of chronic pancreatitis would provide valuable insight into the natural history of IRAP. Our findings lend support to the belief that recurrent pancreatitis may be an intermediate stage in the development of chronic pancreatitis in many patients. ${ }^{18,19}$

The reliability of diagnosis of chronic pancreatitis on EUS has been investigated by many groups.Studies comparing EUS findings with histology have shown EUS to have a sensitivity of about $90 \%$ in detecting chronic pancreatitis. ${ }^{20-22}$ EUS has good correlation with endoscopic pancreatic function tests. ${ }^{5,6,23}$ Hence EUS has the potential to detect chronic pancreatitis at early stages without the disadvantage of being very invasive (histology) or cumbersome (pancreatic function tests). ${ }^{24}$ The diagnostic yield of EUS may improve in the future with newer advances like digital image analysis. ${ }^{11}$ EUS relies on many diagnostic criteria and interobserver differences with regard to individual features may affect the diagnostic outcome. Previous studies have shown that while there may be disparity with regards to individual features among observers, there is good agreement among them for the overall diagnosis of chronic pancreatitis. ${ }^{10,25}$

Our study has certain limitations. As biliary sphincter manometry was not performed in our patients, some of our patients with IRAP may be having sphincter of Oddi dysfunction and may not be truly idiopathic., . $^{316}$ Performing biliary sphincter manometry is technically demanding and has a significant risk of complications. This test was not done in many series reporting patients with recurrent pancreatitis. ${ }^{4,17}$ EUS may not detect all cases of pancreas divisum and some patients with this condition may have been missed; this is alsoa limitation of magnetic resonance imaging. ${ }^{11}$ Tests for genes predisposing to pancreatitis were not done in our study subjects. Usage of Multiple criteria and different cut offsfor diagnosing chronic pancreatitis complicates the ability to efficiently compare results with similar studies. ${ }^{11}$ We chose $>5$ criteria for diagnosing chronic pancreatitis to minimise the number of false positive cases but some other groups have used $>4$ or even $>3$ criteria to diagnose chronic pancreatitis. ${ }^{11,20}$ The development of a single uniform set of criteria is essential to avoid confusion. The Rosemont criteria proposed in 2009 is an important step forward. ${ }^{26}$ Our study includes many patients who underwent EUS well before the Rosemont criteria were proposed and hence we have used the conventional criteria. MRCP was used sparingly, as EUS provided adequate anatomical information while reducing costs.

In conclusion, EUS is an important modality for evaluation of patients with recurrent acute pancreatitis. A significant number of patients with IRAP have underlying chronic pancreatitis and this information may be useful in determining prognosis, management strategies andscheduling follow up and surveillance.

\section{References}

1. Testoni PA, Caporuscio S, Bagnolo F, Lella F. Idiopathic recurrent pancreatitis: long-term results after ERCP, endoscopic sphincterotomy, or ursodeoxycholic acid treatment. Am J Gastroenterol 2000;95(7):1702-7.

2. Sajith KG, Chacko A, Dutta AK. Recurrent acute pancreatitis: clinical profile and an approach to diagnosis. Dig Dis Sci 2010;55(12):3610-6.

3. Coyle WJ, Pineau BC, Tarnasky PR, et al. Evaluation of unexplained acute and acute recurrent pancreatitis using endoscopic retrograde cholangiopancreatography, sphincter of Oddi manometry and endoscopic ultrasound. Endoscopy 2002;34(8):617-23.

4. Yusoff IF, Raymond G, Sahai AV. A prospective comparison of the yield of EUS in primary vs. recurrent idiopathic acute pancreatitis. Gastrointest Endosc 2004;60(5):673-8.

5. Albashir S, Bronner MP, Parsi MA, Walsh RM, Stevens T. Endoscopic ultrasound, secretin endoscopic pancreatic function test, and histology: correlation in chronic pancreatitis. Am J Gastroenterol 2010;105(11):2498-503.

6. Stevens T, Dumot JA, Parsi MA, Zuccaro G, Vargo JJ. Combined endoscopic ultrasound and secretin endoscopic pancreatic function test in patients evaluated for chronic pancreatitis. Dig Dis Sci 2010;55(9):2681-7.

7. Li ZS. Progress in endoscopic management of pancreas diseases. World J Gastroenterol 1998;4(2):178-80.

8. Bhutani MS, Hoffman BJ, Hawes RH. Diagnosis of pancreas divisum by endoscopic ultrasonography. Endoscopy 1999;31(2):167-9.

9. Levy MJ, Geenen JE. Idiopathic acute recurrent pancreatitis. Am J Gastroenterol 2001;96(9):2540-55.

10. Wiersema MJ, Hawes RH, Lehman GA, Kochman ML, Sherman S, Kopecky KK. Prospective evaluation of endoscopic ultrasonography and endoscopic retrograde cholangiopancreatography in patients with chronic 
abdominal pain of suspected pancreatic origin. Endoscopy 1993;25(9):555-64.

11. Romagnuolo J. EUS in inflammatory diseases of the pancreas. In Endosonography (second edition) Elsevier publishing 2011:127-47.

12. Lara LF, Levy MJ. Idiopathic recurrent acute pancreatitis. MedGenMed 2004;6(4):10.

13. Petrone MC, Arcidiacono PG, Testoni PA. Endoscopic ultrasonography for evaluating patients with recurrent pancreatitis. World J Gastroenterol 2008;14(7):1016-22.

14. Morris-Stiff G, Webster P, Frost B, Lewis WG, Puntis MC, Roberts SA. Endoscopic ultrasound reliably identifies chronic pancreatitis when other imaging modalities have been non-diagnostic. Jop 2009;10(3):280-3.

15. Talamini G, Bassi C, Butturini G, et al. Outcome and quality of life in chronic pancreatitis. Jop 2001;2(4):11723.

16. Fischer M, Hassan A, Sipe BW, et al. Endoscopic retrograde cholangiopancreatography and manometry findings in 1,241 idiopathic pancreatitis patients. Pancreatology 2010;10(4):444-52.

17. Norton SA, Alderson D. Endoscopic ultrasonography in the evaluation of idiopathic acute pancreatitis. Br J Surg 2000;87(12):1650-5.

18. Guda NM, Romagnuolo J, Freeman ML. Recurrent and relapsing pancreatitis. Curr Gastroenterol Rep 2011;13(2):140-9.

19. Mariani A, Testoni PA. Is acute recurrent pancreatitis a chronic disease? World J Gastroenterol 2008;14(7):995-8.

20. Varadarajulu S, Eltoum I, Tamhane A, Eloubeidi MA. Histopathologic correlates of noncalcific chronic pancreatitis by EUS: a prospective tissue characterization study. Gastrointest Endosc 2007;66(3):501-9.

21. Chong AK, Hawes RH, Hoffman BJ, Adams DB, Lewin DN, Romagnuolo J. Diagnostic performance of EUS for chronic pancreatitis: a comparison with histopathology. Gastrointest Endosc 2007;65(6):808-14.

22. Bhutani MS, Arantes VN, Verma D, et al. Histopathologic correlation of endoscopic ultrasound findings of chronic pancreatitis in human autopsies. Pancreas 2009;38(7):8204.

23. Conwell DL, Zuccaro G, Purich E, et al. Comparison of endoscopic ultrasound chronic pancreatitis criteria to the endoscopic secretin-stimulated pancreatic function test. Dig Dis Sci 2007;52(5):1206-10.

24. Hernandez LV, Catalano MF. EUS in the diagnosis of early-stage chronic pancreatitis. Best Pract Res Clin Gastroenterol 2010;24(3):243-9.

25. Wallace MB, Hawes RH, Durkalski V, et al. The reliability of EUS for the diagnosis of chronic pancreatitis: interobserver agreement among experienced endosonographers. Gastrointest Endosc 2001;53(3):294-9.

26. Catalano MF, Sahai A, Levy M, et al. EUS-based criteria for the diagnosis of chronic pancreatitis: the Rosemont classification. Gastrointest Endosc 2009;69(7):1251-61. 